\title{
A Fast-Acquisition PLL using Split Half-Duty Sampled Feedforward Loop Filter
}

\author{
Woo-Yeol Shin, Manho Kim, Gi-Moon Hong, and Suhwan Kim, Senior Member, IEEE
}

\begin{abstract}
We reduce the pattern jitter and acquisition time of a phase-locked loop (PLL) by adopting the split halfduty sampled feedforward loop filter. A prototype designed and fabricated in a $0.18 \mu \mathrm{m}$ standard CMOS technology has a $40 \%$ lower acquisition time than a PLL without operating in fast acquisition mode. Its peak-to-peak jitter is $26 \%$ less than that of a PLL with a conventional 2nd-order RC loop filter. ${ }^{l}$
\end{abstract}

Index Terms - Acquisition time, loop filter, pattern jitter, phase locked loop (PLL).

\section{INTRODUCTION}

Phase-locked loops are key components of integrated circuits and consumer electronics products, providing a stable clock source to digital, analog circuits and systems. Timing jitter is inherent in PLLs, and this needs to be minimized if circuits are to operate properly. In a charge-pump PLL, the main source of jitter, excluding the inherent oscillator phase noise and any supply noise, is the pattern jitter caused by the non-ideal nature of the charge pump. Several techniques have been proposed to reduce pattern jitter [1], [2]. In particular, a half-duty sampled feedforward loop filter can achieve a low pattern jitter, and it only requires a simple control method, and imposes no circuit overhead [2].

In recent consumer electronics products, PLLs are commonly turned off when the system enters sleep mode, in order to reduce overall power consumption. This makes it important that the output clock of the PLL has a short acquisition time when emerging from the sleep mode. This can be achieved by gear-shifting techniques which change the charge-pump current or the capacitance of the loop filter [3]-[5]. However, these techniques are required to change at least two of PLL parameters because their techniques are based on 3rd-order PLL. This requires a complicated architecture which takes up a lot of area. To solve this problem, in this paper, we present a new design of PLL circuit adopting the split half-duty sampled feedforward loop filter.

The remainder of this paper has four sections. Section II provides an overview of the pattern jitter problems. Section III describes our new PLL architecture. In Section IV, we present

\footnotetext{
${ }^{1}$ This work was supported by the IT R\&D program of MKE/KEIT [Development of Standard Technology for Next Generation Semiconductor Devices and Equipments].

W.-Y. Shin, G.-M. Hong, and S. Kim are with the Electrical Engineering and Computer Sciences, Seoul National University, Seoul, 151-742, Korea (email:suhwan@snu.ac.kr).

M. Kim is with Samsung Electronics, Yong-In 446-711, Korea.
}

measured results from an experimental prototype PLLs, designed and fabricated using a $0.18 \mu \mathrm{m}$ CMOS technology. Section V concludes the paper and summarizes the potential benefits of our approach.

\section{The Problem of Pattern Jitter}

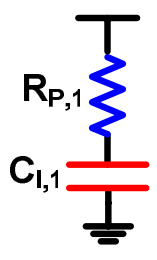

(a)

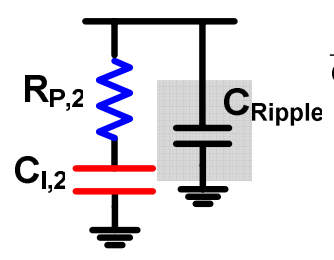

(b)

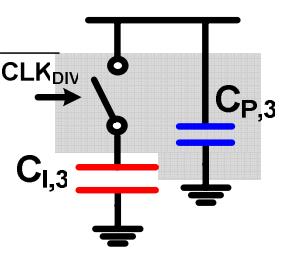

(c)

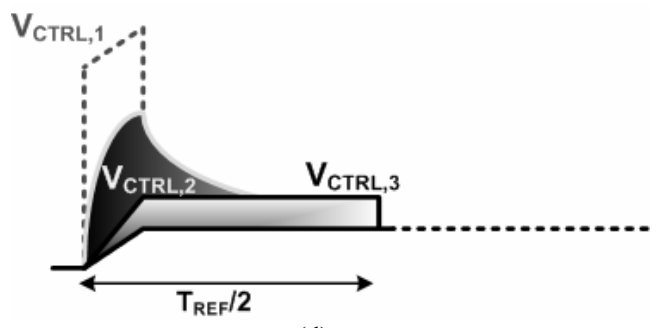

(d)

Fig. 1. Loop filter networks and the ripple of VCTRL:, (a) 1st-order RC loop filter, (b) 2nd-order RC loop filter, (c) half-duty sampled feedforward loop filter and (d) ripple of VCTR.

Charge-pump PLL needs both proportional gain and integral gain for stable feedback. In a conventional charge-pump PLL, as shown in Fig. 1(a), a resistor in the loop filter makes proportional gain and a capacitor makes integral gain. However, the resistor causes a lot of ripple in $\mathrm{V}_{\mathrm{CTRL}}$ of the voltage-controlled oscillators (VCO), as shown in Fig. 1(d). This ripple causes frequency fluctuations in the VCO and pattern jitter in the PLL. In many designs, this ripple in $\mathrm{V}_{\mathrm{CTRL}}$ is filtered by an additional capacitor, $\mathrm{C}_{\text {Ripple, }}$, as shown in Fig. 1(b). However, the insertion of $\mathrm{C}_{\text {Ripple }}$ tends to degrade the stability of the PLL, and careful design is required to ameliorate this problem. Moreover, filtering $\mathrm{V}_{\text {CTRL }}$ with $\mathrm{C}_{\text {Ripple }}$ does not totally eliminate fluctuations in $\mathrm{V}_{\mathrm{CTRL}}$. To alleviate this problem, proportional gain spreading techniques have been proposed [1], [2]. By spreading the proportional gain across either a full reference period or half of a reference period, these feedforward loop filters dramatically reduce pattern jitter. In particular, as shown in Fig. 1(c), a half-duty sampled feedforward loop filter has a straightforward 
architecture, a simple control method, and almost no overhead. A half-duty sampled feedforward loop filter works in the following way: First, it samples the charge from the charge pump using the proportional capacitor $\mathrm{C}_{\mathrm{P}, 3}$, and the voltage across $\mathrm{C}_{\mathrm{P}, 3}$ achieves the proportional gain. Then, when $\mathrm{CLK}_{\mathrm{DIV}}$ is low, the sampled charge in $\mathrm{C}_{\mathrm{P}, 3}$ is shared with the integral capacitor $\mathrm{C}_{\mathrm{I}, 3}$. Eventually, this shared voltage produces integral gain. A half-duty sampled feedforward loop filter does not rely on $\mathrm{C}_{\text {Ripple }}$ to provide filtering, but acts as a $1^{\text {st }}$-order RC loop filter (Fig. 1 (a)), with the following characteristics:

$$
\begin{aligned}
& R_{P, 1}=\frac{T_{R E F}}{2} \cdot \frac{1}{C_{P, 3}} \\
& C_{I, 1}=C_{P, 3}+C_{I, 3}
\end{aligned}
$$

We can also show that a half-duty sampled feedforward loop filter has less effect on stability than a $2^{\text {nd }}$-order RC loop filter shown in Fig.1(b), because the additional pole in the open-loop transfer function that would be created by $\mathrm{C}_{\text {Ripple }}$ is eliminated.

\section{Proposed LoOP Filter}

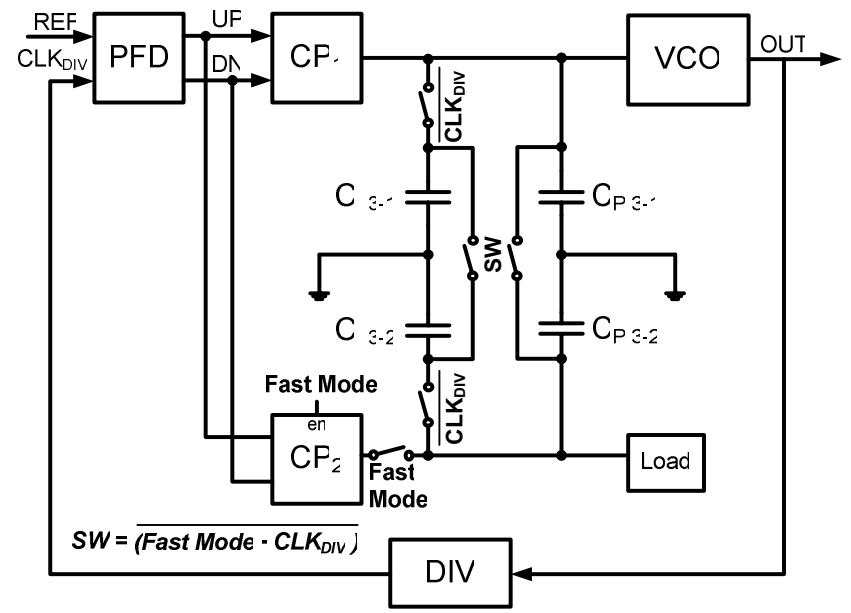

Fig. 2. Block diagram of the proposed PLL with a split half-duty sampled feedforward loop filter.

Figure 2 shows PLL architecture with proposed split halfduty sampled feedforward loop filter. Because this type of loop filter has $1^{\text {st }}$-order characteristics, we do not have to adjust several parameters to achieve a sufficient phase margin [4]. Instead, we split the capacitances $C_{I}$ and $C_{P}$ of the halfduty sampled feedforward loop filter into two equal parts.

Our new loop filter operates as follows: In fast acquisition mode, both $\mathrm{CP}_{1}$ and $\mathrm{CP}_{2}$ turn on transfer charge into the split loop filter. In the sampling phase of the fast acquisition mode, when $\mathrm{CLK}_{\mathrm{DIV}}$ is high, charge from $\mathrm{CP}_{1}$ is sampled by $\mathrm{C}_{\mathrm{P}, 3-1}$, while charge from $\mathrm{CP}_{2}$ is simultaneously sampled by $\mathrm{C}_{\mathrm{P}, 3-2}$. After $\mathrm{CLK}_{\mathrm{DIV}}$ goes low, sharing phase of the fast acquisition mode starts. Charge sampled at $\mathrm{C}_{\mathrm{P}, 3-1}$ and $\mathrm{C}_{\mathrm{P}, 3-2}$ is shared across all the capacitors: $\mathrm{C}_{\mathrm{P}, 3-1,1}, \mathrm{C}_{\mathrm{P}, 3-2,} \mathrm{C}_{\mathrm{I}, 3-1}$, and $\mathrm{C}_{\mathrm{I}, 3-2}$. This step makes our design tolerant of any mismatch in size between the upper and lower capacitors. As $\mathrm{C}_{\mathrm{P}, 3-1}$ and $\mathrm{C}_{\mathrm{P}, 3-2}$ are charged at the same time, and $\mathrm{C}_{\mathrm{I}, 3-1}$ and $\mathrm{C}_{\mathrm{I}, 3-2}$ are sharing their charge, the effective capacitance of the PLL during fast acquisition mode can be expressed as follows:

$$
\begin{aligned}
& C_{P, \text { fast }}=C_{P, 3-1}=C_{P, 3-2}=\frac{C_{P, 3}}{2} \\
& C_{I, \text { fast }}=C_{I, 3-1}=C_{I, 3-2}=\frac{C_{I, 3}}{2}
\end{aligned}
$$

In a charge-pump PLL, the acquisition time mainly consists of pull-in time and lock time [6]. The pull-in time of a PLL is not explained by an s-domain linear model or by s-domain loop transfer analysis. Pull-in time is only related to the large signal behavior of the PLL, and follows this equation:

$$
T_{\text {pull_in }} \propto \frac{I_{C P}}{C_{I, 1}}=\frac{I_{C P}}{C_{I, 3}+C_{P, 3}}
$$

However, the lock-time of a PLL exactly depends on its loop bandwidth in the s-domain linear model:

$$
T_{\text {lock }} \propto B W=\frac{K_{V C O} I_{C P} R_{P, 1}}{2 \pi \cdot N} \propto R_{P, 1} \propto \frac{1}{C_{P, 3}}
$$

When our PLL is in fast acquisition mode, the effective capacitances of both $C_{P}$ and $C_{I}$ are halved, reducing both pullin time and lock time by half. This allows the system to wakeup quickly from sleep mode.

When the PLL gets frequency acquistion, it is switched from fast acquisition mode to normal operation mode. In normal operation mode, $\mathrm{CP}_{2}$ is turned off and the charge from $\mathrm{CP}_{1}$ is transferred to both $\mathrm{C}_{\mathrm{P}, 3-1}$ and $\mathrm{C}_{\mathrm{P}, 3-2}$. Then, the sampled charge is shared across all the capacitors. Therefore, the equivalent loop filter capacitances are as follows:

$$
\begin{aligned}
& C_{P, \text { normal }}=C_{P, 3-1}+C_{P, 3-2}=C_{P, 3} \\
& C_{I, \text { normal }}=C_{I, 3-1}+C_{I, 3-2}=C_{I, 3}
\end{aligned}
$$

In normal operation mode, the loop bandwidth of the PLL stabilizes, and the magnitude of the fluctuation in $\mathrm{V}_{\mathrm{CTRL}}$ is reduced because of the larger capacitance of the loop filter.

\section{EXPERIMENTAL RESULTS}

The proposed PLL was designed and fabricated in a standard $0.18 \mu \mathrm{m}$ CMOS process. Its microphotograph is shown in Fig. 3. To assess the relative jitter, a PLL with $2^{\text {nd }}-$ order loop filter were also designed and fabricated on the same die. 


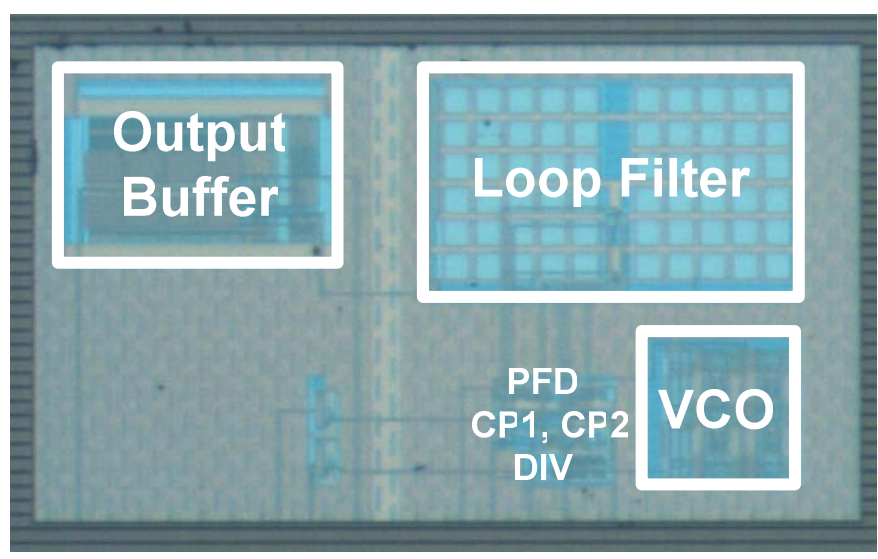

Fig. 3. Die photograph of the proposed PLL.
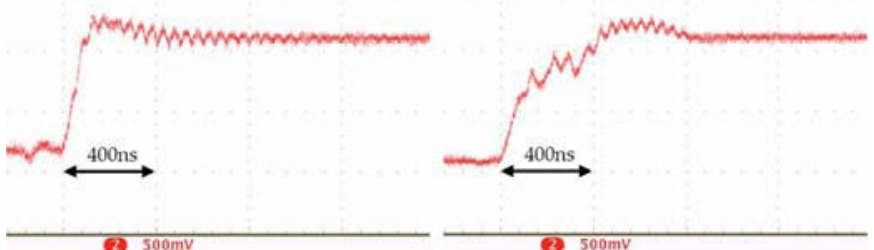

Fig. 4. Measured control voltage of PLL: a split half-duty sampled feedforward loop filter in (a) fast acquisition mode and (b) normal operation mode.

Figure 4 shows measured control voltage of PLL to check the acquisition time of the proposed PLL in both fast acquisition mode and normal operation mode. The reference frequency in normal operation is $20 \mathrm{MHz}$, the output frequency is $1.6 \mathrm{GHz}$. The measurement results suggest that the acquisition time in fast acquisition mode is about $40 \%$ less than in normal operation mode.

TABLE I

COMPARISON OF JITTER PERFORMANCE

\begin{tabular}{|c|c|c|}
\hline Symbol & $\begin{array}{c}\text { RMS } \\
\text { Jitter (ps) }\end{array}$ & $\begin{array}{c}\text { Peak-to-Peak } \\
\text { Jitter (ps) }\end{array}$ \\
\hline $\begin{array}{c}\text { Conventional } \\
2^{\text {nd }} \text {-order RC loop filter }\end{array}$ & 4.173 & 30.40 \\
\hline $\begin{array}{c}\text { Split half-duty sampled } \\
\text { feedforward loop filter } \\
\text { (fast acquisition mode) }\end{array}$ & $\mathbf{5 . 4 1 8}$ & 40.00 \\
\hline $\begin{array}{c}\text { Split half-duty sampled } \\
\text { feedforward loop filter } \\
\text { (normal operation mode) }\end{array}$ & $\mathbf{3 . 0 7 6}$ & $\mathbf{2 2 . 4 0}$ \\
\hline
\end{tabular}

Table 1 and Figure 5 show the measured jitter performance of the PLL. The RMS jitter of our PLL is 3.076ps, and the peak-to-peak jitter is 22.40ps. We see that our PLL in its normal operation mode has lower pattern jitter than a conventional 2nd-order RC loop filter, and almost the half amount of jitter as a PLL in the fast acquisition mode.

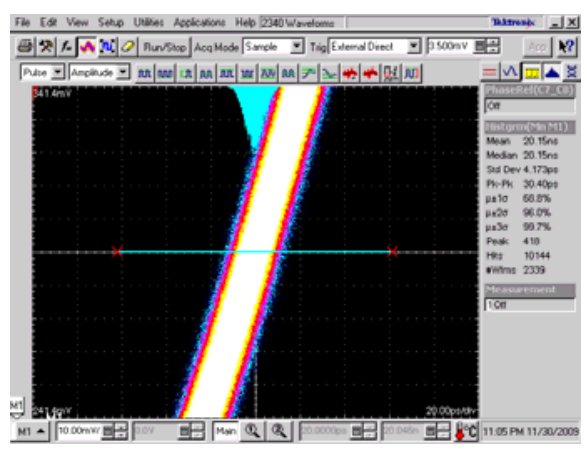

(a)

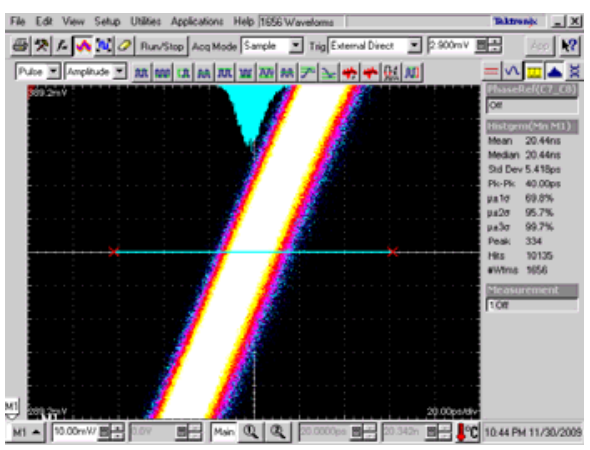

(b)

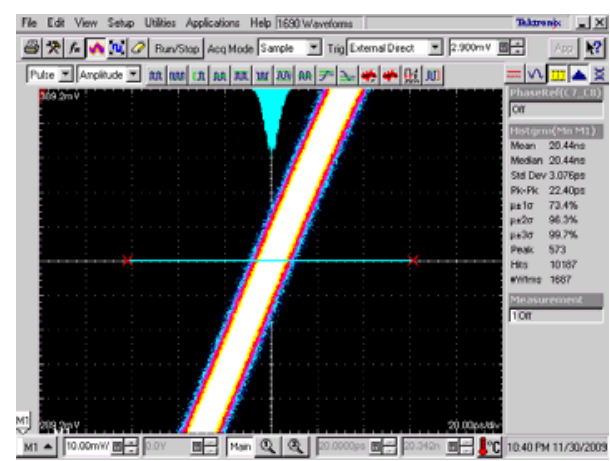

(c)

Fig. 5. Measured jitter histogram: (a) 2nd -order RC loop filter, (b) a split half-duty sampled feedforward loop filter in fast acquisition mode and (c) a split half-duty sampled feedforward loop filter in normal operation mode.

\section{Conclusion}

A PLL with our split half-duty sampled feedforward loop filter can achieve a fast acquisition time, by effectively reducing the capacitance of the loop filter as well as a low pattern jitter, by spreading the proportional gain during half of the reference cycle. The PLL is silicon-proven in a standard $0.18 \mu \mathrm{m}$ CMOS technology, and its performance was experimentally assessed by measuring the acquisition time as well as jitter characteristics. The IC has an active area of $0.068 \mathrm{~mm}^{2}$ and consumes $10.28 \mathrm{~mW}$ at a $1.8 \mathrm{~V}$ supply. 


\section{REFERENCES}

[1] J. G. Maneatis, J. Kim, I. McClatchie, J. Maxey, and M. Shankaradas, "Self-biased high-bandwidth low-jitter 1-to-4096 multiplier clock generator PLL," IEEE J. Solid-State Circuits, vol. 38, no. 11, pp. 1795 -1803 , Nov. 2003.

[2] J. Kim, J.-K. Kim, B.-J. Lee, N. Kim, D.-K. Jeong, and W. Kim, “A 20$\mathrm{GHz}$ phase-locked loop for $40 \mathrm{~Gb} / \mathrm{s}$ serializing transmitter in $0.13 \mu \mathrm{m}$ CMOS," in Proc. Symp. VLSI Circuits Dig., pp. 144-147, June 2005.

[3] D. Byrd and C. Davis, "A fast locking scheme for PLL frequency synthesis," Application Notes, National Semiconductor Corporation, Jul 1995.

[4] J.-K. Woo, D.-K. Jeong, and S. Kim, "Fast-locking CDR circuit with autonomously reconfigurable mechanism," IEE Electronics Letters, vol. 43, no. 11, pp. 624-626, May 2007.

[5] Y. Sumi. S. Obote, K. Narai, K. Tsuda, K. Syoubu and Y. Fukui, "Fast settling PLL frequency synthesizer utilizing the frequency detector method speedup circuit," IEEE Trans. Consumer Electron., vol. 43, no. 3, pp. 550-558, Aug. 1997.

[6] S. I. Ahmed and R. D. Mason, "Improving the acquisition time of a PLL-based, integer-N frequency synthesizer," in Proc. Int. Symp. on Circuits and Systems, pp. 365-368, May 2004,.

\section{BIOGRAPHIES}

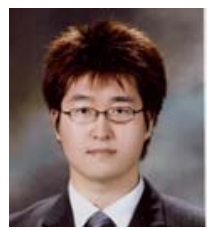

Woo-Yeol Shin received the B.S. degree in electrical engineering from Seoul National University, Korea, in 2005 . He is currently working toward the Ph.D. degree in electrical engineering at Seoul National University. His research interests include high-speed I/O circuits and high-speed memory interfaces.

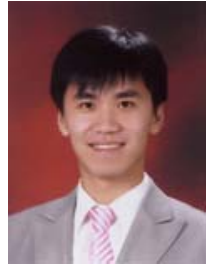

Manho Kim received the B.S. and M.S. degrees in electrical engineering from Seoul National University, Seoul, Korea, in 2007 and 2010, respectively. He is currently with Samsung Electronics, Co., Ltd. His research interests are related to the design of analog and mixed signal integrated circuits.

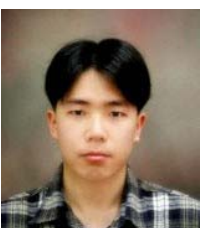

Gi-Moon Hong received the B.S. degree in electrical engineering from Seoul National University, Seoul, Korea, in 2009 . Since 2009 , He is currently pursuing MS degree at the same university. His research interests include high speed serial link circuits.

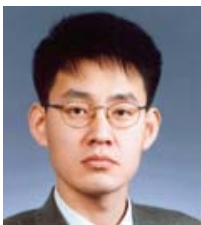

Suhwan Kim (M'01-SM'07) received the B.S. and M.S. degrees in electrical engineering and computer science from Korea University, Seoul, Korea, in 1990 and 1992, respectively and the Ph.D. degree in Electrical Engineering and Computer Science from the University of Michigan, Ann Arbor MI, in 2001.

From 1993 to 1999, he was with LG Electronics, Seoul, Korea. From 2001 to 2004, he was a Research Staff Member in IBM T. J. Watson Research Center, Yorktown Heights NY.

In 2004, Dr. Kim joined Seoul National University, Seoul, Korea, where he is currently an Associate Professor of Electrical Engineering. His research interests encompass high-performance and low-power analog and mixed signal integrated circuits and technology, digitally compensated analog and $\mathrm{RF}$ circuits, high-resolution time-to-digital converters, and high-speed I/O circuits. 